\title{
Distribution, Amplification, and Summation of Cyclic Nucleotide Sensitivities within Single Olfactory Sensory Cilia
}

\author{
Hiroko Takeuchi and Takashi Kurahashi \\ Graduate School of Frontier Biosciences, Osaka University, Toyonaka, Osaka 560-8531, Japan
}

\begin{abstract}
Submicron local cAMP elevation was used to map the distribution of transduction channels in single olfactory cilia. After the fine fluorescent visualization of the cilium with the laser-scanning confocal microscope, the intraciliary cAMP was jumped locally with the laser beam that photolyzes cytoplasmic caged compounds. Simultaneously, cells' responses were obtained with the whole-cell patch clamp. Responses were observed anywhere within the cilia, showing the broad distribution of transduction channels. For odor detection, such distribution would be useful for expanding the available responding area to increase the quantum efficiency. Also, the stimulus onto only $1 \mu \mathrm{m}$ region induced $>100 \mathrm{pA}$ response operated by $>700 \sim 2300$ channels, although only $1 \mathrm{pA}$ is sufficient for olfactory cells to generate action potentials. The large local response indicates a presence of strong amplification achieved with a high-density distribution of the transduction channels for the local ciliary excitation.
\end{abstract}

Key words: signal transduction; channel; cAMP; calcium; olfaction; vision

\section{Introduction}

Olfaction starts from the activation of receptor protein located at the sensory cilia (Buck and Axel, 1991) that display a nano-scale tubing structure (100 200 nm diameter). Receptor activation drives enzymatic cascades involving G-protein and the adenylyl cyclase (AC) system that increases cytoplasmic cAMP molecules in the cilia (Pace et al., 1985; Sklar et al., 1986; Jones and Reed, 1989; Lowe et al., 1989; Bakalyar and Reed, 1990). These chemical reactions are in turn converted into electrical signals by two ion channels: cyclic nucleotide-gated (CNG) cationic channels and $\mathrm{Ca}^{2+}$-activated $\mathrm{Cl}^{-}\left(\mathrm{Cl}_{(\mathrm{ca})}\right)$ channels (Kurahashi, 1989; Firestein et al., 1990; Gold et al., 1990; Kleene and Gesteland, 1991; Lowe and Gold, 1991; Kurahashi and Yau, 1994; Firestein and Shepherd, 1995; Schild and Restrepo, 1998; Gold, 1999; Frings et al., 2000; Firestein, 2001). Thus, the outline of the signal conversion has been now fairly well established. Quantification of this system is being conducted, but the nanotube structure of the cilium prevents some of fundamental experiments.

In fact, the basic question even for the spatial distribution of the systems is still controversial. In the early electrophysiological study using the ciliary bundle, Lowe and Gold (1991) showed that cyclic nucleotide $(\mathrm{CN})$ sensitivities are distributed evenly along entire cilia. With the electron microscopy (EM), however, immunostaining against the subunit of $\mathrm{CNG}$ channel, CNGA2, was

\footnotetext{
Received Aug. 3, 2007; revised Nov. 28, 2007; accepted Dec. 3, 2007.

This work was supported by grants from the Human Frontier Science Program (T.K.), Ministry of Environment (T.K.), the Research Foundation for Opto-Science and Technology (T.K.), Ministry of Education, Culture, Sports, Science, and Technology (H.T., T.K.), and Shiseido Science Research Grant (H.T.). We thank Dr. Yoshihiro Kubo for his valuable comments throughout the whole work, Dr. Seiki Kuramitsu for advice on the polyacrylamide gel usages, and anonymous reviewers for comments leading to the greater improvements.

Correspondence should be addressed to Hiroko Takeuchi, Graduate School of Frontier Biosciences, Osaka University, Toyonaka, 0saka 560-8531, Japan. E-mail: hiroko@bpe.es.osaka-u.ac.jp.

DOI:10.1523/JNEUROSCI.3531-07.2008

Copyright $\odot 2008$ Society for Neuroscience $\quad$ 0270-6474/08/280766-10\$15.00/0
}

found to be dominant to the tip of the cilia (Matsuzaki et al., 1999). Recently, Flannery et al. (2006) used the detached insideout ciliary preparation and cAMP diffusion model, and suggested that CNG channels were clustered at the middle part of the cilia.

These controversies arose mainly from the technical limitations in a direct measurement of $\mathrm{CN}$ sensitivities in living cilia. In the present study, we addressed to this question by using a local uncaging of cytoplasmic caged compound with nano-scale UVlaser stimulation that was combined with simultaneous electrical recordings. The single cilium was finely visualized with the laserscanning confocal microscope (LSM) system after loading with the fluorescent compound, Lucifer yellow. When the CN sensitivities were mapped with local UV spots along the single cilium, cells responded to stimuli at any point of the cilia. Furthermore, data will show that local responses are independent from each other in small responses, establishing a linear response summation. At the same time, strong stimulus onto only a very small region $(1 \mu \mathrm{m})$ was found to induce a huge inward current exceeding $100 \mathrm{pA}$, that was equivalent to the opening of $700 \sim 2300$ ion channels. The large local response indicates a presence of strong amplification operated by a high density distribution of the transduction channels for the local excitation.

\section{Materials and Methods}

Preparation and recording. Olfactory receptor cells (ORCs) were dissociated enzymatically $(0.1 \%$ collagenase $)$ from the olfactory epithelium of the newt (Cynops pyrrhogaster) and mouse (Jcl:ICR, 6 weeks old or older) as described previously (Takeuchi and Kurahashi, 2005). The experiments were performed under the latest ethical guidelines for animal experimentation at Osaka University, which is based on international experimental regulations. The mucosae were incubated $\left(5 \mathrm{~min}, 37^{\circ} \mathrm{C}\right)$ in a $\mathrm{Ca}^{2+}$ and $\mathrm{Mg}^{2+}$-free solution containing (in $\mathrm{mM}$ ) $110 \mathrm{NaCl}, 3.7 \mathrm{KCl}, 10$ HEPES, 15 glucose, 1 pyruvate, and $0.001 \%$ phenol red, with $\mathrm{pH}$ adjusted to 7.4 with $\mathrm{NaOH}$, and cells were mechanically isolated. Visualization and recordings were done in the normal Ringer's solution contining (in 
mм) $110 \mathrm{NaCl}, 3.7 \mathrm{KCl}, 3 \mathrm{CaCl}_{2}, 1 \mathrm{MgCl}_{2}, 10$ HEPES, 15 glucose, 1 pyruvate, $0.001 \%$ phenol red, with $\mathrm{pH}$ adjusted to 7.4 with $\mathrm{NaOH}$. We selected ORCs whose cilia attached firmly to the bottom of the culture dish coated with $0.1 \%$ concanavalin A. Sometimes, floating and waving cilia were included in the laser beam, but, such data were clearly recognized by their inconsistency. Data from those cells were omitted from the analysis. Experiments were performed at room temperature $\left(23-25^{\circ} \mathrm{C}\right)$.

Ciliary currents were recorded with the whole-cell (WC) recording configuration (Hamill et al., 1981) under the voltage-clamp mode as described previously (Takeuchi and Kurahashi, 2005). Culture dish was mounted on the inverted microscope (Axiovert; Zeiss, Oberkochen, Germany). Before the experiments, the cell shape without fluorescence was monitored on a personal computer display through a digital camera (D2X; Nikon, Tokyo, Japan). Patch pipettes (resistance 10-15 M $\Omega$ ) were made of borosilicate tubing with filament (World Precision Instruments, Berlin, Germany) by using a two-stage vertical patch electrode puller (PP-830; Narishige, Tokyo, Japan). The recording pipette was, unless otherwise indicated, filled with a Cs solution containing (in mM) 119 $\mathrm{CsCl}, 1 \mathrm{CaCl}_{2}$, 5 EGTA, 10 HEPES, and $0.001 \%$ phenol red, pH 7.4 adjusted with $\mathrm{CsOH}$ containing $0.01 \%$ Lucifer yellow and $1 \mathrm{~mm}$ caged cAMP or $1 \mathrm{~mm}$ caged cGMP (Calbiochem, La Jolla, CA). Data were sampled with pClamp version 8 or 9 (Molecular Devices, Sunnyvale, CA) at $20 \mathrm{kHz}$, after low-pass filtered at $2 \mathrm{kHz}$. Laser stimuli and the data acquisition were regulated from computers synchronized with the TTL pulses generated by pClamp. For curve drawings by using Microcal Origin 7.5 software (OriginLab, Northampton, MA), data were low-pass filtered at $0.02 \mathrm{kHz}$.

To visualize a single cilium filled with the Lucifer yellow, LSM (510; Zeiss) system equipped with Plan-Neofluar (phase contrast) $40 \times / 0.75$ numerical aperture (NA), Plan-Apochromat $63 \times / 1.4$ NA (oil immersion), Fluar (differential interference contrast) $100 \times / 1.3 \mathrm{NA}$ (oil immersion) objective lens was used. Among them, the $63 \times$ Plan-Apochromat lens provides the highest quality of images, but the transmission to UV lens is extremely low (almost $0 \%$ at $351 \mathrm{~nm}$ and $~ 5 \%$ at $364 \mathrm{~nm}$ ). Instead, $100 \times$ Fluar lens has relatively lower quality image, but has a high UV transmission ( $78 \%$ at $351 \mathrm{~nm}$ and $82 \%$ at $364 \mathrm{~nm}$ ). We used these lenses depending on the necessity of the individual experiments.

Because cells were deteriorated drastically during imagings and recordings, we conducted a series of experiments within $10 \mathrm{~min}$ after the establishment of WC recording configuration.

Local photolysis of caged compounds. Caged cAMP or cGMP was initially dissolved in DMSO and then diluted by Cs solution. As reported previously (Takeuchi and Kurahashi, 2002), we did not recognize difference between cAMP and cGMP, except that cGMP had a slight stronger effect on the cilia. With the WC configuration, the cell interior was filled with the pipette solution containing the compounds by free diffusion. The UV laser beam ( $80 \mathrm{~mW}$, Argon laser $\lambda=351,364 \mathrm{~nm}$; Coherent, Santa Clara, CA) was used for photolysis. Through the experiments, UV intensity was regulated by the transmission parameter of an acoustooptic tunable filter (AOTF) device (100\% is full transmission to the particular wavelength). For the local and spatially restricted stimulation, region of interest (ROI) function of the LSM system was used. On the ROI function, the On-Off and position of the UV spot were regulated by the AOTF and a Galvano-mirror, respectively. ROIs were irradiated with so-called "raster scan" by the laser-scanning system, which moves from left to right in parallel to the $x$-axis first. At the edge of ROI, AOTF switches the laser beam on and switches off at the opposite edge. The laser position in turn moves one-step-down to the next Y-position. Because of such sequences, actual laser irradiation is an assembly of the very brief pulse events. At the $4 \mu \mathrm{s} /$ pixel scan speed, for instance, $10 \times 10$ pixels rectangle ROI is irradiated only $400 \mu \mathrm{s}$ in total (see Results) (see Fig. 1). In the present study, we selected either circle or square shape for stimulating area. When the square is used, irradiated length of the cilia is dependent on the passing angle. This was taken into the account for data interpretation. The cell images in the figures display the precise orientations with which experiments were actually conducted.

In some experiments, local ciliary areas were damaged after local UV irradiation, especially with strong UVs. Ciliary damages were recognized from the observation that the restricted part was swelled, or the fluores- cent image was disappeared beyond the point, presumably because of plugging of the cytoplasmic space. We omitted the data from such experiments. Incidentally by such examples, however, we could confirm the selected ROI position to be identical exactly to the ciliary area that received UV irradiations.

During the experiments, timing and duration of the actual laser irradiation were detected by a UV-photodiode (G6262; Hamamatsu Photonics, Hamamatsu, Japan). To guide the laser beam to the photodiode, we used the optical fiber ( $1 \mathrm{~mm}$ diameter) in the system. The tip of fiber was situated near the focal plane with an $\sim 45^{\circ}$ angle. Because the sensitivity of G6262 via the optical fiber for the UV region was still low, a 488 $\mathrm{nm}$ laser was added for detection. The $488 \mathrm{~nm}$ laser beam alone did not cause photolysis when examined with cells. The time resolution of the detection system [a simple current-voltage $(I-V)$ converter with an operational amplifier, LM358; National Semiconductor, Santa Clara, CA] was $<1 \mu$ s that covered the scan speed of $4.48 \mu$ s/pixel used here. Rather, the sampling frequency $(20 \mathrm{kHz})$ became the rate-limiting step for the signal detection, when the small ROIs were selected (e.g., $<10$ pixels for $x$-axis). We corrected for the actual period of the UV stimulation, based on the detected light signals and the shape of the ROIs.

\section{Results}

\section{Measurement of laser spot size}

In this study, one of the important factors was to obtain a small laser spot for the positioned local photolysis of cytoplasmic caged compound. For this, we used LSM-ROI (see Materials and Methods) allowing bleaching at arbitral regions on the focal plane. Laser beams of 351/364 (photolysis), 458 (visualization) and 488 (irradiation detection) nm were used for individual purposes.

It is generally assumed that the laser light at the focal plane expresses roughly a Gaussian distribution as follows (Fig. 1 A,B):

$$
y=\frac{1}{\sigma \sqrt{\frac{\pi}{2}}} e^{-2 \frac{x^{2}}{\sigma^{2}}},
$$

where $x$ is the distance and $\sigma$ is the SD. First of the experiments, we calibrated the size of the light spot at the focal plane to obtain $\sigma$ value of the UV spot. In the LSM-ROI, the total power of UV irradiation is expressed as time and spatial integration of light intensity during the raster scan for the $x-y$ axes continuously (Fig. $1 C)$. Because the laser on and off are repeated while the beam position moves $x-y$ axes surrounded by ROI, light signals were detected as an assembly of pulse events (Fig. $1 D$ ). When the square ROI having $10 \mathrm{Y}$-pixels was selected, nine light pulses were obtained from the photodiode sensor (Fig. 1D). Pulse detection depended on the scan speed and ROI size and, in other words, analog/digital conversion $(20 \mathrm{kHz})$ could not follow high-speed and small-area scanning. In the figures, we represent the time course of laser irradiation as a total period as in Figure $1 D$. Information about the number of pixels surrounded by ROI is indicated in the individual legends.

To calibrate the actual UV irradiation in our system, we monitored bleaching of Lucifer yellow $(0.2 \%)$ that was immobilized with polyacrylamide gel (15\%). A rectangular ROI was selected and was irradiated (458 nm) for 1-30 times (Fig. 1E). Relationship between the number of scan and the bleach showed a linear function before saturation (Fig. $1 F$ ). When the bleaching degree of the boundary part was plotted against the $x$-axis (Fig. $1 G$ ), the relationship was fitted by the error (erf) function that represents the integral of Gaussian function (as can be expected from Fig. $1 A, H)$ :

$$
y=\left(1+\operatorname{erf} \frac{x-a}{\sigma \sqrt{2}}\right) \times \frac{b}{2},
$$


where $a$ and $b$ are constants. The best fit was obtained with $\sigma=0.72 \mu \mathrm{m}$ with a $40 \times$ objective lens (NA, 0.7) and $0.35 \mu \mathrm{m}$ with a $63 \times$ oil-immersion objective lens (NA, 1.4). Both values are nearly consistent with the fact that the spot size $(D)$ is proportional to 1/NA. Because the image for bleaching was obtained from the LSM slicing, the boundary region included the information from a certain thickness. Also, because the UV beam spreads over gradually in proportion to the distance from the focal plane, the numbers obtained here will be the upper limitation.

If we just simply assume that the spot size is proportional to the wavelength $(\lambda)$, $\sigma=0.54 \mu \mathrm{m}$ with a $40 \times$ lens for $364 \mathrm{~nm}$ laser spot and $0.28 \mu \mathrm{m}$ with a $63 \times$ lens. Also, $0.3 \mu \mathrm{m}$ was obtained for a $100 \times$ lens (NA, 1.3) by calculation. Because the total power of UV irradiation was not flat at the focal plane, in the following figures we represent the UV intensity and the stimulus positions with color-gradients that were estimated from these parameters. Throughout the figures, it should be noted that the color-graded area is larger than the ROI.

\section{Visualization and local stimulation}

With the $458 \mathrm{~nm}$ laser excitation of cytoplasmic Lucifer yellow, the ciliary shape was clearly identified to display the entire length from the proximal part to the tip (length, mean $\pm \mathrm{SD}=12 \pm 4 \mu \mathrm{m} ; n=58$ newts) (Fig. $2 B, E, H$ ). In some cases, the proximal part was not visualized, when the knob and/or ciliary bottom did not attach to the coverslip, and when the focal plane was set at the bottom. The ciliary diameter of the fluorescent images was bigger than the EM observation (Lindow and Menco, 1984), obviously because of diffraction of fluorescent light in our experiments. The cilia became thinner at the distal part, as has been reported in the EM studies (Lindow and Menco, 1984).

After the confirmation of the ciliary shape, a small ROI was selected so as to include a particular part of the single cilium (Fig. $2 B, E, H)$. When the stimulation was applied to the ROI with the laser scan (Fig. $2 B, E, H$, arrow), an inward current was obtained under the whole-cell voltage-clamp recordings $\left(V_{\mathrm{h}}=-50 \mathrm{mV}\right)$ (Fig. $2 C, F, I)$. With the $1 \mu \mathrm{m}$ circle ROI $(63 \times, 364 \mathrm{~nm}$ laser only, $100 \%$ transmission), the current amplitude was $5.2 \pm 6.1 \mathrm{pA}$ $(n=38)$. With the raster scan, as mentioned in the Materials and Methods (Fig. 1), the actual illumination is only a very small fraction of the entire stimulus period. Therefore, the small amplitude does not reflect the UV laser power itself to be weak. Also, during the stimulus of several hundreds of milliseconds, the adaptation system equipped in the olfactory cilia may have been developed, resulting to the slight reduction in the response amplitude.

Also, with a raster scan illumination with internal gaps, cytoplasmic cAMP is expected to increase in pulsate ways. One may imagine that the cAMP locally uncaged can be rapidly diffuse along the longitudinal axis of the internal cilia. However, the data (Fig. 2C) show that the current develops in additive ways during the stimulus period. This may indicate that the uncaged cAMP
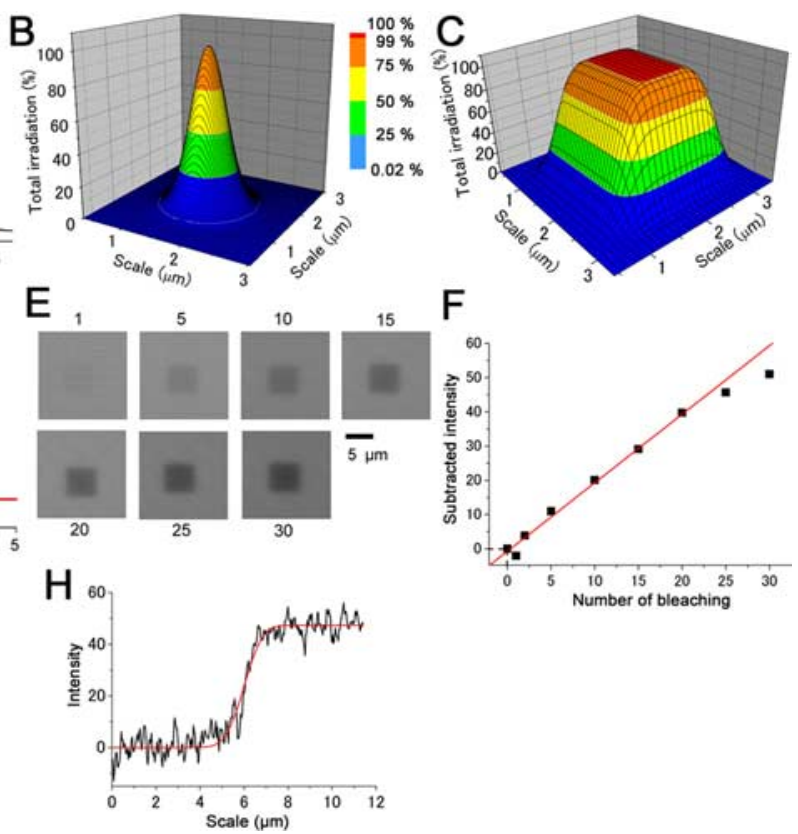

Figure 1. Properties of laser beam and their spatial and time integration during the raster scan. $\boldsymbol{A}$, The laser beam having lized with polyacrylamide gel. $40 \times$ objective lens ( $\lambda=488 \mathrm{~nm} ; 1-30$ times photobleach reactions). The side of the $R 0$ l was 50 列 for 16 line sweeps of the $x$ axis. $\boldsymbol{H}$, Rising phase of the ROl edge fitted by the error function (Eq. 2). The best fitting was obtained with $\sigma=0.54$ at $364 \mathrm{~nm}$ with a $40 \times$ objective lens. Data are from 17 times irradiations.

molecules stay for some time in the vicinity of the produced point. This matter, relating to the diffusion of the cytoplasmic factors, will be further examined below.

With a high UV intensity using the Fluar $100 \times$ lens (note that the Fluar lens has a higher transmission rate for UV components), the current amplitude became large, exceeding $100 \mathrm{pA}$ in response to $1 \mu \mathrm{m}$ spot stimulation (Fig. $2 F$ ). With $100 \mathrm{pA}$, we could roughly calculate the number of ion channels underlying this transduction current. In calculation, the current was estimated to be a mixture of $50 \% \mathrm{CNG}$ and $50 \% \mathrm{Cl}$ components, referring to the whole-cell measurement on the newt (Kurahashi and Yau, 1993). Using the single-channel current, driving force and open probability of $\mathrm{CNG}$ and $\mathrm{Cl}_{(\mathrm{ca})}$ channel, we estimated the number of channels opened to be 200 and 2100 with parameters obtained from the frog (Larsson et al., 1997), 100 and 570 from rat parameters (Reisert et al., 2003), respectively. In total, it was assumed that $\sim 700 \sim 2300$ channels were activated by this local stimulation. As for such a big current with a small spot, one may simply consider that it is generated by the one-dimensional bilateral diffusion of cAMP molecule within the cilium. But it was not the case, as examined further later.

\section{Fundamental properties of responses induced by the local UV spot}

Because the ciliary responses to the local spot were observed for the first time in the present study, we investigated fundamental properties of the responses to confirm whether it is really the cNMP (cyclic nucleotide mono phosphate)-induced response. 

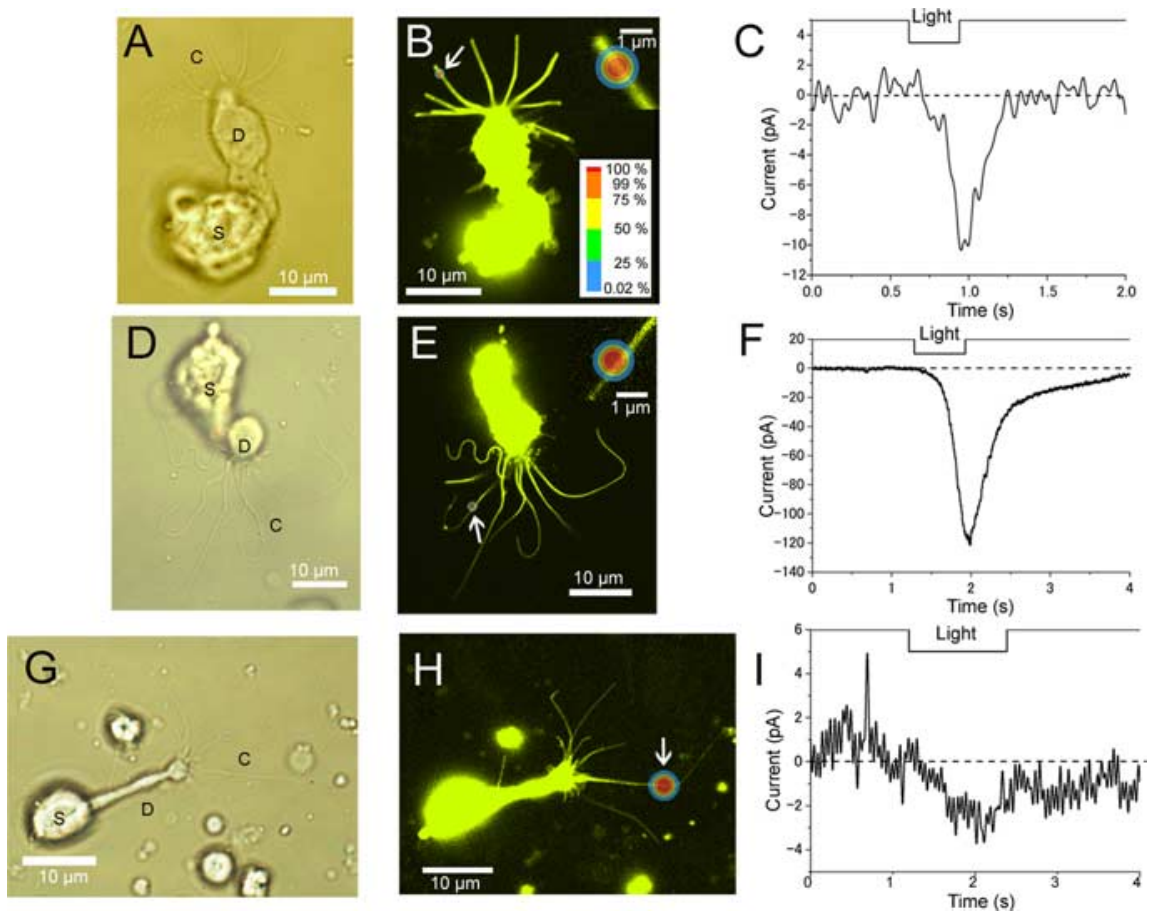

Figure 2. Visualization, local stimulation and responses of the cilia. $\boldsymbol{A}-\boldsymbol{I}$, Cells were obtained from the newt $(\boldsymbol{A}-\boldsymbol{C}, 63 \times$ lens; $\mathbf{D}-\boldsymbol{F}, 100 \times$ lens) and the mouse $(\mathbf{G}-\mathbf{I}, 63 \times$ lens). To visualize the fine cilia, the picture contrast was enhanced with Photoshop. C, Cilia; D, dendrite; S, soma. $\boldsymbol{B}, \boldsymbol{E}, \boldsymbol{H}$, Fluorescent images of ORCS (excitation; $458 \mathrm{~nm}$ ). Stimulated point is indicated with a colored circle and white arrow. Diameter of ROls are 0.98 ( 14 pixels), 1 ( 25 pixels), and 2.94 ( 42 pixels) $\mu \mathrm{m}$, respectively. The inset shows magnified images including the stimulation point. $C, F, I$, Whole-cell current induced by local light stimulation. Cells were loaded with $1 \mathrm{~mm}$ caged $\mathrm{CAMP}(\boldsymbol{F})$ or caged $\mathrm{CGMP}(\boldsymbol{C}, I) . V_{\mathrm{h}^{\prime}},-50 \mathrm{mV}$. Downward deflection of the upper traces indicate the timing and duration of the light stimulation ( $\boldsymbol{C}, \boldsymbol{I}, \lambda=364,488 \mathrm{~nm} ; \boldsymbol{F}, \lambda=351,364,488 \mathrm{~nm}$; output $65 \%$; transmission $100 \%$ ).

$<1$ s, current summation was observed (Takeuchi and Kurahashi, 2003). In the panel, the plot was eliminated to avoid showing complex figures. As for the response reduction, possibilities for the full and local depletion of the caged compound are unlikely, because cAMP loading of the ORC from the whole-cell pipette takes less than a second after the rupture of the patch membrane (see Kurahashi, 1990).

It is well established that this adaptation is mainly regulated by $\mathrm{Ca}^{2+}$ influx through the CNG channel. The cytoplasmic $\mathrm{Ca}^{2+}$ in turn makes Ca-calmoduline (Ca-CAM) complex to suppress $\mathrm{CNG}$ channel in a negative feedback way. If cytoplasmic $\mathrm{Ca}^{2+}$ and resultant $\mathrm{Ca}-\mathrm{CAM}$ complex were freely diffusing within the cilium, the recovery time should become faster with the local stimulus (Fig. $4 E$ ) than the value obtained previously with broad stimulus. Therefore, the result of Figure $4 E$ may indicate that $\mathrm{Ca}^{2+}$ and Ca-CAM works only at the small area with little diffusion. $\mathrm{Ca}^{2+}$ binding site ( $\mathrm{Cl}_{(\mathrm{Ca})}$ channels), $\mathrm{Ca}^{2+}$ buffers (by $\mathrm{Ca}^{2+}$-binding proteins), $\mathrm{Na}^{+}$dependent $\mathrm{Ca}^{2+}$-extrusion system (Reisert et al., 2003), and tight and permanent binding of CAM to the CNG channel (Bradley et al., 2004) may explain some of such diffusion limitation (see below).

These experiments confirmed us that the responses induced by local spots are in-

Mainly, three properties were focused on: dose dependence, voltage dependence, and adaptation.

The response amplitudes were dependent on the laser light intensity (Fig. 3A). With a fixed ROI and scan speed (4.48 $\mu \mathrm{s} /$ pixel), an increase in the light intensity (adjusted with "transmission parameter" for 351/364 nm laser) increased the current amplitude monotonically (Fig. 3B). Because the intensity dependence was obtained with changes on the 351/364 nm laser, again, we could exclude the possibility that $488 \mathrm{~nm}$ laser has a side effect. With the small ROI, the current response did not reach to the saturating level, presumably because of the fast scanning. However, slow scanning will make the interpretation quite complex, because of the cNMP diffusion along the cilia (see below and Discussion).

Next, we examined the voltage dependence. At negative potentials, the current polarity was inward (Fig. $3 C$ ), and reversed at $+5 \mathrm{mV}(5.0 \pm 5.6 \mathrm{mV}, n=5)$ (Fig. 3D). The reversal potential and the shape of the $I-V$ relationship were similar to the previous reports (Kurahashi, 1989; Lowe and Gold, 1993; Takeuchi and Kurahashi, 2002, 2003).

Finally, we examined adaptation of the responses induced by the local simulation. The double UV stimulation caused a reduction in the amplitude in the secondary response as reported for the adaptation of odorant- and CAMP-responses. Because the interstimulus interval was prolonged, the secondary current response recovered gradually (Fig. $4 A-C$ ). Eighty percent recovery was observed at $\sim 5 \mathrm{~s}$ (Fig. $4 D, E$ ). This value is similar to that in previous works (Kurahashi and Shibuya, 1990; Zufall et al., 1991; Kurahashi and Menini, 1997; Takeuchi and Kurahashi, 2003; Takeuchi and Kurahashi, 2005). When interstimulus interval was deed induced by the local increase of the cytoplasmic cAMP concentration $\left([\mathrm{cAMP}]_{\mathrm{i}}\right)$.

\section{Spatial distribution of $\mathrm{CN}$ sensitivities along the single cilium} When $\mathrm{CN}$-sensitivities were mapped with local stimuli by changing the ROI position along the cilium (Fig. 5A), the current was observed wherever the UV spot stimulates (Fig. 5B-G). We saw big responses at the proximal part of the cilia, but they are interpreted to be caused by the stimulus onto the multiple cilia. Because of this observation, however, it is assumed that the functional CNG channels are located also at the proximal part of the cilia. Therefore, it is likely that cNMP sensitivities are distributed broadly along the entire cilium (Fig. $5 H, I$ ). The same experiments were done on 18 cells ( 13 newts and five mice) with essentially the same results. We never saw the biggest response at the distal part. In both the newt and mouse, large responses were observed when the knob was stimulated. It has been reported that $\mathrm{CNG}$ and $\mathrm{Cl}_{(\mathrm{Ca})}$ channels are present also at the knob (Kaur et al., 2001; Reisert et al., 2003). In the present work, however, we did not make an additional analysis for examining the origin of the current observed when the knob was stimulated.

One may pay particular attention to the fact that the amplitude of the responses gradually decreases as the position moves to the distal part. Similar tendency has been reported by Flannery et al. (2006). It seems unlikely that the reduction is caused by the change in the sensitivity of CNG channel or diffusion limitation of caged substances (i.e., concentration of caged compound is lower at the distal tip), because intensity-response relationship showed a vertical shift when examined between distal and proximal parts in the same cilium (Fig. $5 J$ ). Essentially the same results 

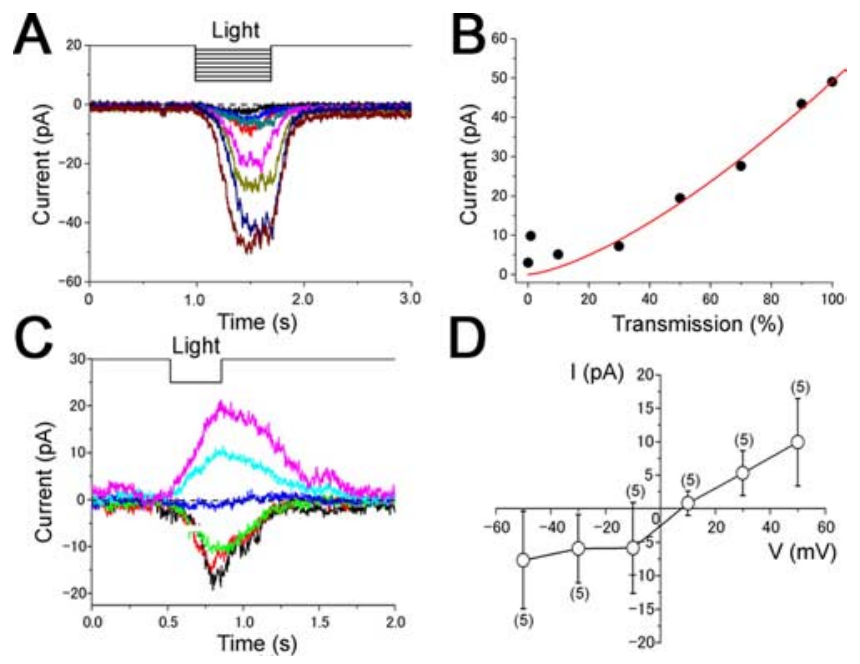

Figure 3. $A-D$, Dose $(A, B)$ and voltage dependence $(\boldsymbol{C}, \boldsymbol{D})$ of the local light-induced responses. Ciliary currents were recorded from a newt ORC loaded with $1 \mathrm{~mm}$ caged CAMP. $\boldsymbol{A}$, Intensity was changed by regulating transmission parameter of LSM: $0.1,1,10,30,50,70,90$, and $100 \%$ ( $100 \times$ lens; $\lambda=351,364 \mathrm{~nm}$; output $65 \%)$. The diameter of the ROI was $1 \mu \mathrm{m}(25$ pixels). $V_{h^{\prime}}-50 \mathrm{mV}$. $B$, Dose-response relationship of the light-induced current. Peak amplitudes of responses obtained in $\boldsymbol{A}$ were plotted against the laser transmission. $C$, Responses were recorded at various holding potential. $V_{\mathrm{h}}=-50$ to $+50 \mathrm{mV}$. The side length of the ROI was $0.98 \mu \mathrm{m}$ ( 14 pixels; $63 \times$ lens; $\lambda=364 \mathrm{~nm}$; output $65 \%$; transmission $100 \%$ ). D, I-V relationship of five cilia; the reversal potential was $5.0 \pm 5.6 \mathrm{mV}$.

were obtained from three different cells. Or, one may think that this is simply caused by the reduction in the across membrane potential depending on the cable theory, especially when we consider that the cilia display an extremely thin structure. The next kind of experiment was conducted to investigate such a possibility.

\section{Summation of small local responses}

In the experiment of Figure 6, we applied two local stimuli independently and simultaneously. If the small and gradual reduction in the current response is caused by the short length constant of the cilium at the resting level, membrane resistance $\left(R_{\mathrm{m}}\right)$ is comparable with intracellular resistance $\left(R_{\mathrm{i}}\right)$. And if we stimulate point $\mathrm{C}$ to reduce $\mathrm{R}_{\mathrm{m}}$ at point $\mathrm{C}\left(R_{\mathrm{mC}}\right)$, it will facilitate the reduction of the across membrane potential at point $\mathrm{B}$ (Fig. $6 \mathrm{~A}$ ). Therefore, the response induced by simultaneous stimulus onto $\mathrm{B}$ and $\mathrm{C}$ will become smaller than the data sum $(\mathrm{B}+\mathrm{C})$ obtained independently (Fig. 6B,C). However, our experimental results clearly showed that responses induced by simultaneous stimulus onto $\mathrm{B}$ and $\mathrm{C}$ became identical to the data summation obtained from independent stimuli (Fig. 6D).

Significance of the resistance drop caused by the local stimulation is justified by referring to the input resistance of the ORCs. The current amplitude of the local UV-induced responses was $\sim 10 \mathrm{pA}$ at $50 \mathrm{mV}$. This number provides $0.2 \mathrm{nS}$ (equivalent to 5 $\mathrm{G} \Omega$ resistance) for the cAMP-induced conductance, only for the $1 \mu \mathrm{m}$ irradiation. The whole-cell input resistance is already $5 \mathrm{G} \Omega$ (Kurahashi, 1989). Furthermore, it has been reported that the change in the across membrane potential along the cilium is extremely small because of the high basal resistance of the cilium (Kleene, 1992). Voltage profiles within the cilium at responses will be investigated below.

The same experiments were performed on five cells. Essentially the same results were obtained from four cells. Unexpectedly, in one case, the data sum $(B+C)$ of independent responses became bigger than the simultaneous stimulation (B and C). At this point we do not know the reason for this phenomenon; this could just be a change in the sensitivity, response drift or slight $\mathrm{Ca}^{2+}$ diffusion and resulted adaptation. The question is remained open, together with the consistency of the results.

With one exception, however, it is strongly suggested that the voltage drop with basal ciliary conductance is small. High basal resistance of the cilia would be responsible for the establishment of a long length constant along the cilia (Kleene, 1992). Furthermore, basal electrogeneity equipped in the olfactory cilia (Kleene, 1992) may be responsible for this. These possibilities must be examined further detail in future. All together, the results thus indicate that the absolute number of channels itself becomes smaller at the tip of the cilia. This may be related to the fact that the distal part is actually thinner than the proximal part (Fig. 6A). Such morphology has been reported for the EM observations (Lindow and Menco, 1984).

When the cilium is situated vertically, namely, when the cilium is situated in parallel to the $y$-axis in the LSM view, two ROI selections could perform the double pulse stimuli with some intervals because of the raster scan (Fig. $6 F, G$ ). With several seconds of interstimulus interval, responses were not influenced with prepulse UV stimulation $(n=3)$ (Fig. $6 F-H)$. The same experiments were also performed on three other cells to which no EGTA was incorporated. Again, the responses were not reduced remarkably after the conditioning pulse. In addition to the aforementioned result of recovery from the adaptation, this result also supports the notion that the diffusion of cytoplasmic factors jumped at restricted part is quite limited (see below).

\section{$\mathrm{CN}$ sensitivities in different cilia}

After visualizing the branching patterns of the cilia from the knob, ROIs were set on three different cilia at approximately equal distances from the knob (Fig. 7A). When each point was stimulated, current responses were induced with almost the same amplitude among different cilia (Fig. $7 B-D$ ). Essentially the same results were observed in six cells. Thus, we conclude that there are cAMP-triggered machineries homogeneously across the cilia.

We also stimulated different cilia with two ROIs simultaneously or independently. The responses did not influence each other. It is likely that the responses are independent, obviously because of the diffusion limitations for the cytoplasmic cAMP and $\mathrm{Ca}^{2+}$ beyond cilia.

\section{Summation of large local responses}

We showed that only a small ROI stimulation caused a big response exceeding $100 \mathrm{pA}$, when the stimulating UV intensity was strong (Fig. $2 F$ ). Summation of such large local responses was examined to understand the behavior of cilia during the natural odorant response, in which entire cilia are stimulated with high dose of $[\mathrm{cAMP}]_{\mathrm{i}}$. First, a single cilium was stimulated with small spots along the longitudinal axis. Next, the entire cilium was stimulated with a long-shape ROI (Fig. 7E). If the local responses were completely independent, the data sum of the individual responses should be identical to the response induced by the total illumination. In our experiments, however, the data sum was bigger than the response induced by the broad illumination (Fig. $7 H)$. The same experiment was conducted in 3 different cells. In two cells the data sum was bigger than the response induced by the large ROI. In one example, both were approximately identical. Even for this particular case, if we take the phosphodiesterase (PDE) effect to be stronger at the local simulation into account, the data sum of local responses seems to be bigger than the simul- 

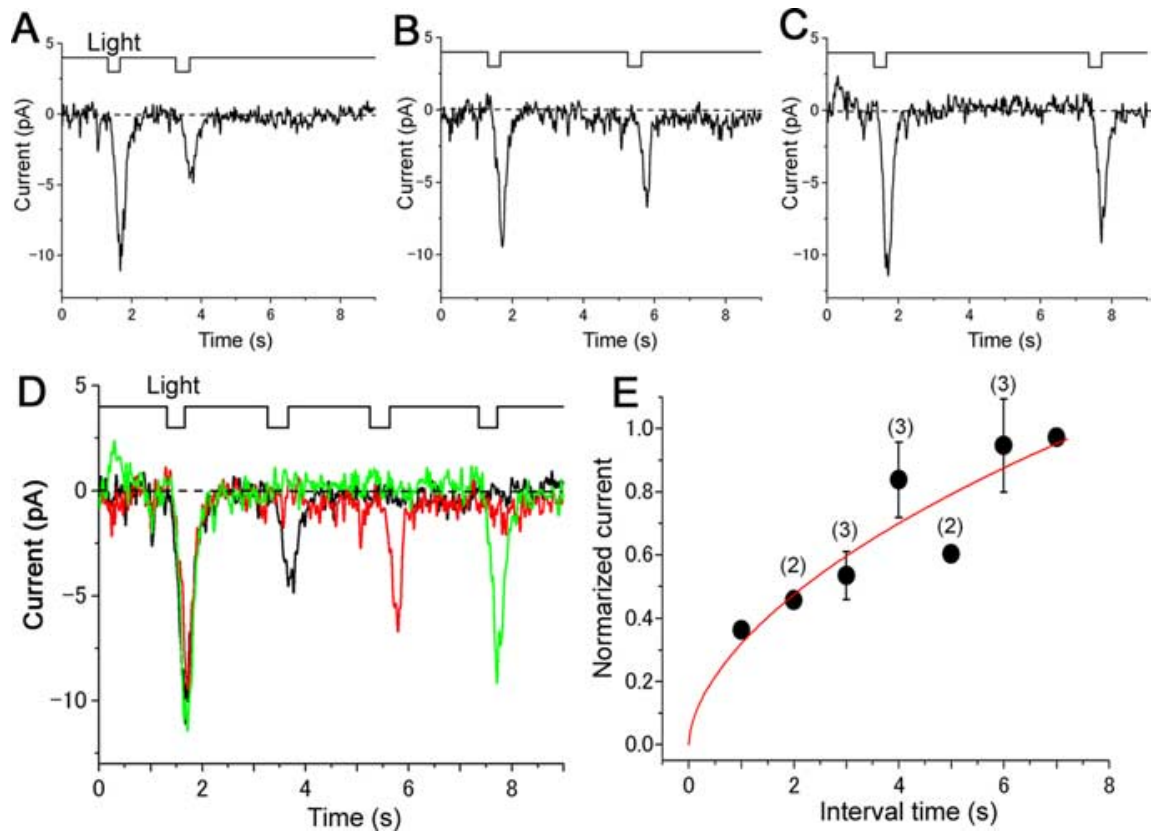

Figure 4. Responses induced by double local photolysis of caged CGMP.A-C, Interstimulus intervals: 2, 4, and $6 \mathrm{~s}$. The side length of the ROI was $0.98 \mu \mathrm{m}$ (14 pixels; $63 \times$ lens; $\lambda=364 \mathrm{~nm}$; output $65 \%$; transmission $100 \%) . V_{\mathrm{h}}=-50 \mathrm{mV}$. D. Recovery from the adaptation. $\boldsymbol{E}$, The smooth line was drawn by a least square fitting by the Hill equation. The cell was obtained from the newt.
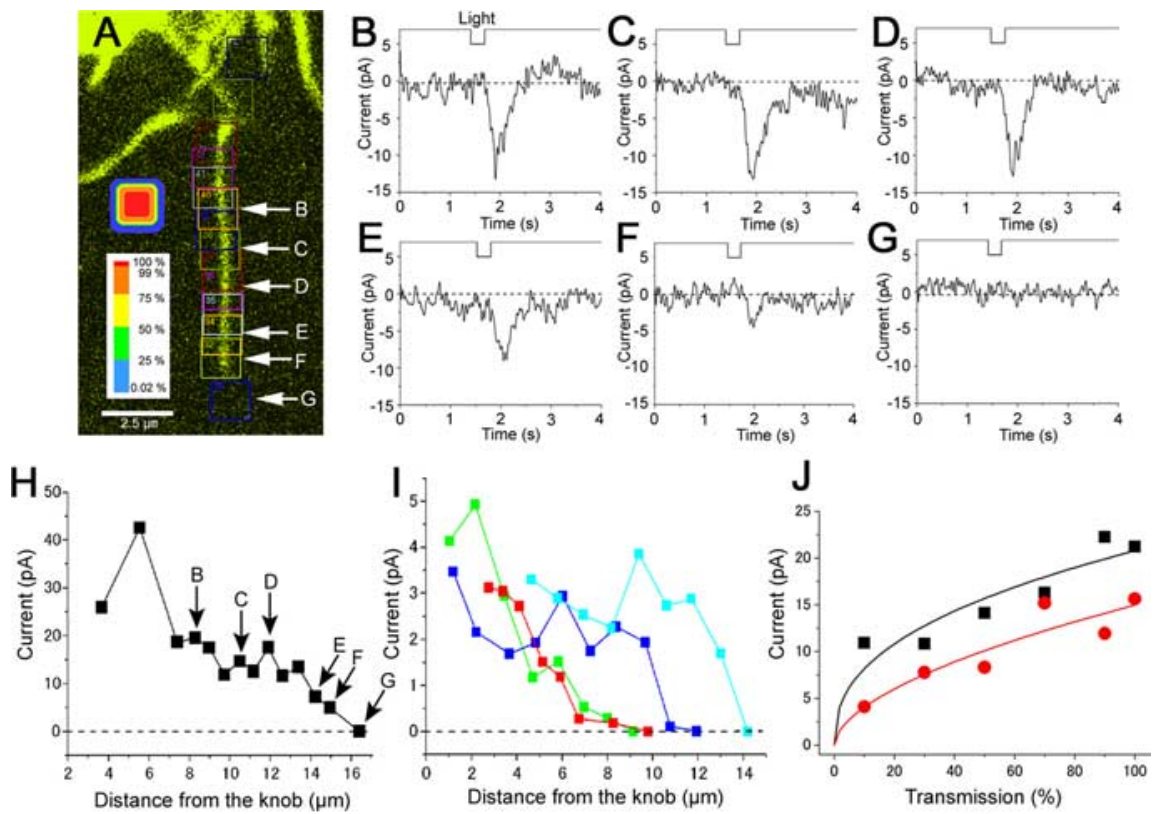

Figure 5. Mapping of $\mathrm{CN}$-sensitivity along the single newt cilium. $\boldsymbol{A}$, Fluorescent image of a single cilium. To avoid complex figure, only ROls are illustrated. Stimulus intensity is shown independently with a colored scale. $\boldsymbol{B}-\boldsymbol{G}$, Waveforms of the current induced by the local laser irradiation. Cell was loaded with $1 \mathrm{~mm}$ caged cGMP. The side length of ROI, $1.47 \mu \mathrm{m}$ (21 pixels; 63X lens; $\lambda=364 \mathrm{~nm}$; output $65 \%$; transmission $100 \%$ ). $V_{h},-50 \mathrm{mV}$. $\boldsymbol{H}$, Relationship between the distance from the knob and local current responses in $\boldsymbol{A}$. I, Relationship between the distance from the knob and local current responses from four cells $(63 \times$ lens). All ROls were circular (diameter was $0.98 \mu \mathrm{m} ; 14$ pixels). Lengths of cilia were variable that is represented as a steep falling down of the response. J, Dose-response relationship at proximal (black) and distal (red) cilia. The diameter of the R0I was $2 \mu \mathrm{m}$ ( 25 pixels; $100 \times$ lens; $\lambda=351$ and 364 nm; output $65 \%$; transmission $100 \%$ ).

taneous stimulation of the cilium. The bigger amplitude of the data sum could be explained by a bilateral (but restricted) diffusion of cytoplasmic factors or reduction in the length constant of the voltage profile which is responsible for the simultaneous excitation of long ciliary region with a large unit conductance. The former possibility was examined with a double pulse protocol with strong spots as in Figure $6 E-H$. The latter, voltage profile within the cilium, will be calculated and examined below.

It has been reported for detached ciliary preparation that the cytoplasmic cAMP diffusion coefficient is $2.7 \times 10^{-6} \mathrm{~cm}^{2} / \mathrm{s}$ (Chen et al., 1999). Therefore, with $x$ (average movement $)=(2 \mathrm{Dt})^{1 / 2}$, the total cilia (20 $\mu \mathrm{m}$ length) could be filled with the cAMP within $1 \mathrm{~s}$. However, experimental data observed here are inconsistent with such simple idea. For instance, double pulse responses onto $2 \mu \mathrm{m}$ distance were completely independent even with a very large response (Fig. $6 E-H$ ). With this distance, therefore, it is unlikely that the cytoplasmic factors (cAMP and/or $\mathrm{Ca}^{2+}$ ) diffuse laterally. One may simply think that this is just because of the long interstimulus interval and complete degradation of cAMP molecules. However, if once CNG channels are open, $\mathrm{Ca}^{2+}$ comes in and makes adaptation that continues for some periods of time (Fig. 4). The fact that we did not see any adaptation at the $2 \mu \mathrm{m}$ distance position (Fig. $6 E-H$ ) suggests that cAMP (and $\mathrm{Ca}^{2+}$ ) diffusion is restricted to less than this distance. Such hindered diffusion of cytoplasmic factors may be explained by the relationship between the total number of molecules, diffusion coefficient, the buffering capacity and the extrusion/degradation rates (see Discussion). Also, in the work of Chen et al. (1999), the $D$ value was dependent on the $[\mathrm{cAMP}]_{\mathrm{i}}$. The value $2.7 \times 10^{-6} \mathrm{~cm}^{2} / \mathrm{s}$ was obtained at $[\mathrm{cAMP}]_{\mathrm{i}}=5 \mu \mathrm{M}$. They suggest that this value becomes smaller when $[\mathrm{cAMP}]_{\mathrm{i}}$ increases. In our previous experiments, $[\mathrm{cAMP}]_{\mathrm{i}}$ was assumed to become as high as $60 \mu \mathrm{M}$ (Takeuchi and Kurahashi, 2005). It thus seems likely that the lateral diffusion of cAMP and $\mathrm{Ca}^{2+}$ is limited to the produced area, when the local cilium was stimulated to produce a limited number of cytoplasmic cAMP molecules. In other words, it is interpreted that the large response induced by the local stimulation is generated with the ion channels in the vicinity of stimulus point.

Of course at this point, however, we cannot exclude the possibility that the cytoplasmic factors diffuse $<2 \mu \mathrm{m}$. Actually, only 1 $\mu \mathrm{m}$ diffusion to both directions from $1 \mu \mathrm{m}$ UV spot will cause the $2 / 3$ reduction in the whole-cilia response in compare with the data sum, when the channels are saturated. This needs to be examined in further.

\section{Electrical length constant at total-cilia responses}

In the previous section it was assumed that the lateral diffusion of cytoplasmic factors was limited to $<2 \mu \mathrm{m}$. Even if the bilateral diffusion was $1 \mu \mathrm{m}$, the observation of $100 \mathrm{pA}$ current with $1 \mu \mathrm{m}$ spot indicates that ORCs produces $>30 \mathrm{pA} / \mu \mathrm{m}$-cilium. We have 
to understand how the cilia behave in total when a high current density is present. Of special interest is if the whole-cell current $(\sim 1 \mathrm{nA})$ can be explained with such a very high density of $30 \mathrm{pA} / \mu \mathrm{m}$ length cilium. Simply, multiplying $30 \mathrm{pA} / \mu \mathrm{m}$ length cilium to $20 \mu \mathrm{m}$ length yields $600 \mathrm{pA}$. A cell having 10 cilia must produce $6 \mathrm{nA}$, but it differs greatly from the real value (Kurahashi, 1989, 1990; Lowe and Gold, 1993).

Figure 8 illustrates simple voltage profiles and current densities derived from the cable theory, with standard parameters (diameter, $0.1 \mu \mathrm{m}$; unit conductance of cytoplasm, $g i=1.7 \mu \mathrm{S} / \mu \mathrm{m}$ ) (Hodgkin and Rushton, 1946; Kleene, 1992). The voltage profile versus the distance from the knob is varied depending on the current density (Fig. $8 \mathrm{~A}$ ). Multiplying these voltage profiles with the unit membrane conductance yields the distribution of current flow within the cilium (Fig. 8B). The curves show that the current amplitude is fairly constant spanning entire cilium when the current density is low, but the length constant becomes shorter as the membrane conductance increases. This indicates that, at weak stimuli by odorants, the olfactory cilia use their entire region. But, as the intensity increases, the total length for current flow is shortened. At $100 \mathrm{pA} / \mu \mathrm{m}$, the current becomes $\sim 10 \%$ at $\sim 6 \mu \mathrm{m}$ distance from the knob. This property may be related to the limitation for the depletion of cytoplasmic $\mathrm{Cl}^{-}$even with the strong odor stimulation.

Integrals of the curves in Figure $8 B$ represent the whole-cell currents as illustrated in Figure $8 C$. The relationship shows a strong sublinear function, explaining a smaller total current than the linear summation of local currents, as the result of Figure $7 \mathrm{H}$. Interestingly, the total ciliary currents do not differ so much between the cilia having 15 and $30 \mu \mathrm{m}$ length. Thus, the ciliary length seems not to be so important especially when the current density is high. This may at least in part explain why the wholecell current is much smaller than the simple summation of local responses. In addition, a voltage drop may occur also at the knob where all ciliary bottoms are connected.

\section{Discussion}

The present study shows that olfactory transduction channels are present broadly on the sensory cilia. The biggest advantage of broad channel expression would be the expansion of available area to the entire ciliary membrane, especially at small responses. In addition, the stimulus onto only a small region $(1 \mu \mathrm{m})$ caused a huge inward current exceeding 100 pA generated by the opening of $>700 \sim 2300$ ion channels. Such large current generation was unexpected, because whole ORCs has a high input impedance exceeding $5 \mathrm{G} \Omega$; only $1 \mathrm{pA}$ of current can generate $5 \mathrm{mV}$ of depolarization that causes action potentials (Lynch and Barry, 1989). The big current was likely to be generated at restricted area, because double pulse stimuli to $2 \mu \mathrm{m}$ distances were almost independent each other. The large local response thus indicates a presence of strong amplification operated by a high density distribution of the transduction channels. This signal amplification has been shown to be nonlinear (Lowe and Gold, 1993; Kurahashi and Menini, 1997; Takeuchi and Kurahashi, 2003; Takeuchi and Kurahashi, 2005), and the basal bias for cytoplasmic cAMP is set at lower point than the threshold. Therefore, it is concluded that individual ORCs behave like as a switching device with an economical system. In such system, however, input signals are distorted during the signal amplification. In the electrical circuit, very similar system has been traditionally termed as class- $C$ amplifier. It is interesting to note, in comparison, that photoreceptor cells that have a homologous G-protein-mediated enzymatic cascade have a high bias point for the cytoplasmic cGMP level. Such system could perform a linear signal amplification without distortion (equivalent to class-A amplifier in the electrical circuit), using a constant and big basal energy supply.

It is well known that rod photoreceptor cells can detect very small light signal, down to the single photon (Baylor et al., 1979). Also, in the rod, CNG channels are distributed evenly across the outer segment. Their high sensitivities are achieved in the enzymatic cascade that has very high molar-amplification. However, it's becoming common that the ORC is not able to detect the signal from the single odorant molecule (Lowe and Gold, 1995). This is because olfactory signal transduction has low amplification system. At the maximum odorant stimulation, cAMP production rate is comparable with that of the single photon event of the rod (Takeuchi and Kurahashi, 2005). Also in this sense ORC's system can be economical.

Nevertheless, recently, Bhandawat et al. (2005) reported that in the $\mathrm{Ca}^{2+}$-free condition ORCs became sensitive, and the elementary events showed bump-like responses, like as responses to single molecules. There are still questions if such bump-like events express the real binding and dissociation transitions of the 

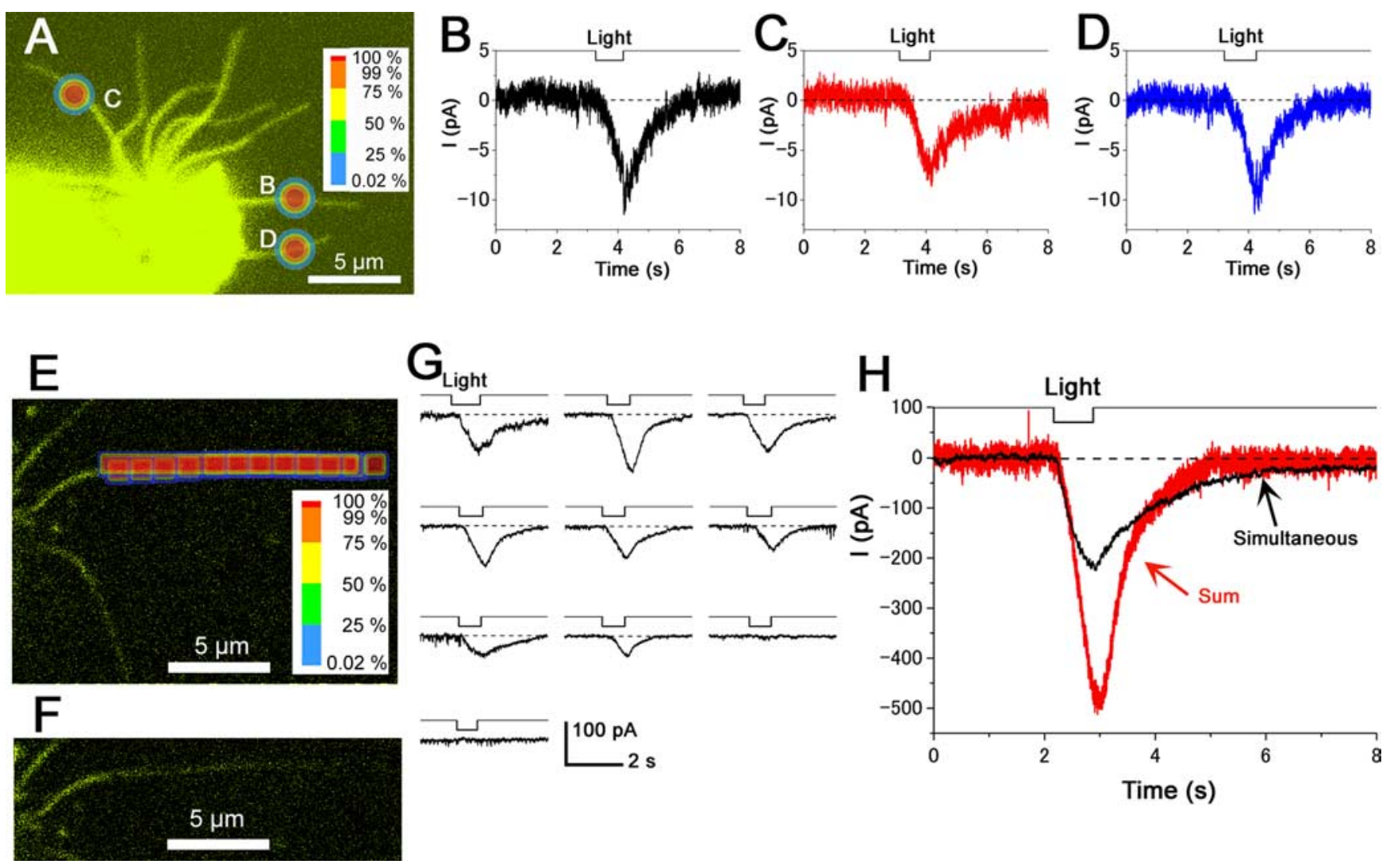

Figure 7. Stimulus with multiple ROls. Cells were loaded with $1 \mathrm{~mm}$ caged CAMP. A, Comparison of $\mathrm{CN}$ sensitivities between cilia. Fluorescent image of a single $\mathrm{ORC}$. The diameter of the ROI was $1.56 \mu \mathrm{m}$ ( 39 pixels). $\boldsymbol{B}-\boldsymbol{D}$, Whole-cell current induced by local light stimulation in different cilia ( $100 \times$ lens; $\lambda=351,364 \mathrm{~nm}$; output $65 \%$; transmission $100 \%) . V_{h^{\prime}}-50 \mathrm{mV}$. $E$, Nonlinear spatial summation of large responses. Fluorescent image of a single newt cilium. ROl position was moved from a proximal part to a distal part. The side length of the small-rectangle ROI was $1 \mu \mathrm{m}$, and the long-rectangle ROI was $1 \times 12 \mu \mathrm{m}(25 \times 300$ pixels; $100 \times$ lens; $\lambda=351,364 \mathrm{~nm}$; output $65 \%$; transmission $100 \%)$. $\boldsymbol{F}$, Single cilium in $\boldsymbol{E}$ without ROI display. $\boldsymbol{G}$, Current responses induced by each small ROI stimuli in $\boldsymbol{E}$. $\boldsymbol{H}$, Comparison of the data sum (red) and response induced by the simultaneous stimulation (black). All data from newt cells.

A

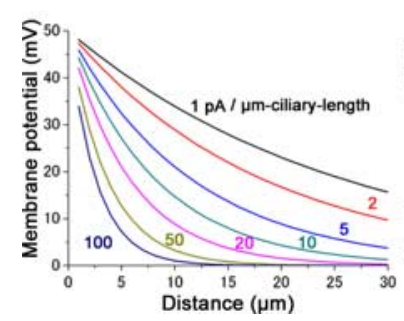

B

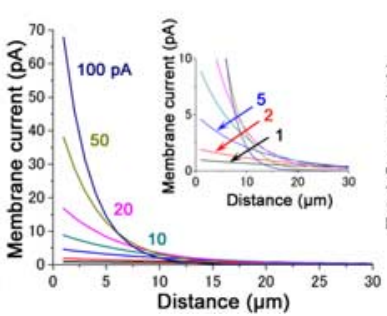

C

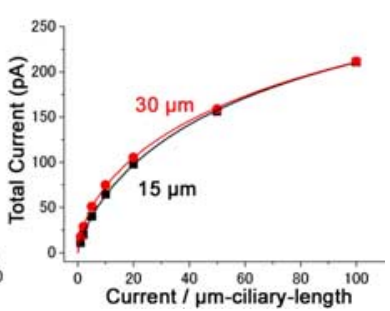

Figure 8. $\boldsymbol{A}-\boldsymbol{C}$, Estimation of the voltage profile $(\boldsymbol{A})$, current density $(\boldsymbol{B})$, and the total ciliary current during the response $(\boldsymbol{C})$. $A$, Relationship between the distance from the knob and membrane potential. Membrane potential was estimated from the cable theory. Parameters: unit conductance, $g i=1.7 \mu \mathrm{S} / \mu \mathrm{m}$; diameter, $0.1 \mu \mathrm{m}$; currents, $1,2,5,10,20$, and $50 \mathrm{pA}$ and $100 \mathrm{pA} / \mu \mathrm{m}$ ciliary length at $50 \mathrm{mV}$. $\boldsymbol{B}$, Relationship between the distance and membrane current. The inset shows magnified data. $\boldsymbol{C}$, Estimation of total current of a single cilium. Length of cilium is $30 \mu \mathrm{m}$ (red) and $15 \mu \mathrm{m}$ (black). Note that the total current is not influenced by the length of cilium especially at large responses.

ligand-receptor interaction. One of the biggest questions is that the data interpretation is based on the assumption that the CNG channel distributes over entire cilia, and that the unitary events are independent each other. There are several reports that CNG channels distribute in a clustering manner within the cilia (Matsuzaki et al., 1999; Flannery et al., 2006). In those cases, unitary events could not be uniform. Our present data, however, provide evidence that single molecule responses can be observed in broad ciliary regions if the basal sensitivities were raised with $\mathrm{Ca}^{2+}$-free media. Furthermore, our data show that local cAMP responses are independent, expressing the linear summation. This is also

consistent with the fact that the unitary event showed discrete amplitudes depending on the number of bound molecules. In addition, the present finding of large response induced by a local stimulation provides a possibility for the generation of single odorant response even in the normal condition (Menini et al., 1995), if there was a particular odorant receptor that has a long lifetime when bound with a certain specific odorants.

With the single cilium electrophysiology and the cAMP diffusion model, Flannery et al. (2006) reported that CNG channels were little expressed at both the distal and proximal parts of the cilia, whereas the channels are clustered at around $20 \%$ position from the proximal part. As for the gradual reduction of CNG current depending on the longitudinal axis, general tendencies were essentially the same between their observations and ours, especially, when one assumes that the absolute distance from the proximal part determines the number of the CNG channels. Because newts have shorter cilia (10-20 $\mu \mathrm{m}$ length) than the frog $(\sim 100 \mu \mathrm{m})$, it is possible that our mapping is applied to the partial region. It is worth to try the same experiment on the animals having longer olfactory cilia. However, our data show that $\mathrm{CN}$ sensitivities are present also at the proximal part of the cilia. Because Flannery et al. (2006) mea- 
sured the CNG channel only, whereas in our experiments we looked at the complex of $\mathrm{CNG}$ and $\mathrm{Cl}_{(\mathrm{ca})}$ components, it is possible, as they raised a possibility, that $\mathrm{Cl}_{(\mathrm{ca})}$ channels may compensate for the spatial deviation. However, signal transmission from CNG current to $\mathrm{Cl}_{(\text {ca) }}$ current is very nonlinear (Lowe and Gold, 1993; Takeuchi and Kurahashi, 2002), such possibility is less likely. In fact, Leinders-Zufall et al. (1997) (their Fig. 7) showed that intraciliary $\mathrm{Ca}^{2+}$ concentration is elevated evenly during the odorant stimulation. To completely solve these questions, direct experiments on the spatial distribution of $\mathrm{Cl}_{(\mathrm{ca})}$ channels in the olfactory cilia using caged Ca would be useful.

The present data may suggest that the diffusion of cytoplasmic factors (cAMP and $\mathrm{Ca}^{2+}$ ) is hindered, when a limited number of the molecules are produced locally. The diffusion of cytoplasmic factors within the thin cylindrical structure is obviously determined by both the buffering capacity and extrusion/degradation systems. It should be noted furthermore that in the nanotube structure the surface/volume ratio is extremely high, providing the strong trapping efficiencies for the soluble molecules by the binding molecules attached to the cytoplasmic membrane surface. In the previous work by Takeuchi and Kurahashi (2005), cytoplasmic cAMP level was estimated to be an order of hundred $\mu \mathrm{M}$ at the maximum that is equivalent to $\sim 500$ molecules $/ \mu \mathrm{m}$ length (calculated from $0.1 \mu \mathrm{m}$ diameter). Density of the CNG channel is $300 / \mu \mathrm{m}$-length (Kurahashi and Kaneko, 1991), and individual CNG channels has four cAMP-binding sites (Varnum and Zagotta, 1996; Zheng and Zagotta, 2004; Chen et al., 2006), providing the total number of cAMP binding sites in $1 \mu \mathrm{m}$ length cilia to be 1200 , which is more than the number of cAMP molecules produced. Single CNG channels show open (bursting) events of $\sim 100 \mathrm{~ms}$, which likely represents the mean lifetime of the cAMP-CNG complex (Kurahashi and Kaneko, 1993). Therefore, the CNG channels will initially trap most of the produced cAMP molecules with a very efficient surface/volume ratio achieved by the nanotube structure, and, simultaneously and subsequently, free cAMP molecules and AMP molecules gradually dissociated from the CNG channels are hydrolyzed by PDE. These processes may, at least in part, explain the outline of the hindered diffusion for the limited number of the local cAMP molecules. Similarly, as for $\mathrm{Ca}^{2+}$ dynamics, several $\mathrm{Ca}^{2+}$. binding molecules $\left[\mathrm{Cl}_{(\mathrm{Ca})}\right.$, CAM, P26olf (proposed by Miwa et al., 2000), and unidentified $\mathrm{Ca}^{2+}$-binding proteins] and $\mathrm{Ca}^{2+}$ extrusion systems ( $\mathrm{Na} / \mathrm{Ca}$ exchange) (Reisert et al., 2003) have been reported, although very little is known for their activities. In addition to the quantification of the related elements, real time and direct imaging of the cytoplasmic factors would be useful for understanding the diffusion processes of the soluble factors within the cilia.

It has been reported that the least-effective odorant concentration is a range of $1 \mu \mathrm{M}$ for a variety of odorant species. This suggests that a single ORC responds to the odorant molecule when $10^{8}$ odorant molecules hit 10 cilia per second (calculated from diffusional flux passing the ellipsoidal geometry) (Lowe and Gold, 1995). Also, Bhandawat et al. (2005) showed that the least effective dose of cineole causes a detectable response when 330 cineole molecules bound to the receptor during $50 \mathrm{~ms}$. Based on these numbers, it is assumed that receptor proteins on the ciliary wall can detect only $0.007 \%$ of odorant molecules hitting the ciliary wall. Such so-called quantum efficiency is extremely small in compare with the photon detection in the rod (>50\%) (Baylor et al., 1979). It is likely that the olfactory receptor protein is expressed in entire cilia, because immunostaining against the receptor protein shows a homogeneous expression (Schwarzenbacher et al., 2005), and because the size of odorant-induced current is almost the same as cAMP-induced response (Takeuchi and Kurahashi, 2002, 2003). Our observation of the broad distribution of the transduction systems strongly suggests that the efficiency of $0.007 \%$ detection is somehow achieved by the increase of the available membrane area responsible for the odorant perception. If the cilia used only a local area, this efficiency would become much smaller.

\section{References}

Bakalyar HA, Reed RR (1990) Identification of a specialized adenylyl cyclase that may mediate odorant detection. Science 250:1403-1406.

Baylor DA, Lamb TD, Yau KW (1979) Responses of retinal rods to single photons. J Physiol (Lond) 288:613-634.

Bhandawat V, Reisert J, Yau KW (2005) Elementary response of olfactory receptor neurons to odorants. Science 308:1931-1934.

Bradley J, Bonigk W, Yau KW, Frings S (2004) Calmodulin permanently associates with rat olfactory CNG channels under native conditions. Nat Neurosci 7:705-710.

Buck L, Axel R (1991) A novel multigene family may encode odorant receptors: a molecular basis for odor recognition. Cell 65:175-187.

Chen C, Nakamura T, Koutalos Y (1999) Cyclic AMP diffusion coefficient in frog olfactory cilia. Biophys J 76:2861-2867.

Chen TY, Takeuchi H, Kurahashi T (2006) Odorant inhibition of the olfactory cyclic nucleotide-gated channel with a native molecular assembly. J Gen Physiol 128:365-371.

Firestein S (2001) How the olfactory system makes senses of scents. Nature 413:211-218.

Firestein S, Shepherd GM (1995) Interaction of anionic and cationic currents leads to a voltage dependence in the odor response of olfactory receptor neurons. J Neurophysiol 73:562-567.

Firestein S, Shepherd GM, Werblin FS (1990) Time course of the membrane current underlying sensory transduction in salamander olfactory receptor neurons. J Physiol (Lond) 430:135-158.

Flannery RJ, French DA, Kleene SJ (2006) Clustering of cyclic-nucleotidegated channels in olfactory cilia. Biophys J 91:179-188.

Frings S, Reuter D, Kleene SJ (2000) Neuronal Ca ${ }^{2+}$-activated Cl- channelshoming in on an elusive channel species. Prog Neurobiol 60:247-289.

Gold GH (1999) Controversial issues in vertebrate olfactory transduction. Annu Rev Physiol 61:857-871.

Gold GH, Nakamura T, Lowe G (1990) Studies on the mechanism of olfactory transduction in vertebrates. Neurosci Res [Suppl] 12:127-134.

Hamill OP, Marty A, Neher E, Sakmann B, Sigworth FJ (1981) Improved patch-clamp techniques for high-resolution current recording from cells and cell-free membrane patches. Pflügers Arch 391:85-100.

Hodgkin AL, Rushton WA (1946) The electrical constants of a crustacean nerve fibre. Proc R Soc Lond Biol Sci 133:444-479.

Jones DT, Reed RR (1989) Golf: an olfactory neuron specific-G protein involved in odorant signal transduction. Science 244:790-795.

Kaur R, Zhu XO, Moorhouse AJ, Barry PH (2001) IP3-gated channels and their occurrence relative to $\mathrm{CNG}$ channels in the soma and dendritic knob of rat olfactory receptor neurons. J Membr Biol 181:91-105.

Kleene SJ (1992) Basal conductance of frog olfactory cilia. Pflügers Arch 421:374-380.

Kleene SJ, Gesteland RC (1991) Calcium-activated chloride conductance in frog olfactory cilia. J Neurosci 11:3624-3629.

Kurahashi T (1989) Activation by odorants of cation-selective conductance in the olfactory receptor cell isolated from the newt. J Physiol (Lond) 419:177-192.

Kurahashi T (1990) The response induced by intracellular cyclic AMP in isolated olfactory receptor cells of the newt. J Physiol (Lond) 430:355-371.

Kurahashi T, Kaneko A (1991) High density cAMP-gated channels at the ciliary membrane in the olfactory receptor cell. NeuroReport 1:5-8.

Kurahashi T, Kaneko A (1993) Gating properties of the cAMP-gated channel in toad olfactory receptor cells. J Physiol (Lond) 466:287-302.

Kurahashi T, Menini A (1997) Mechanism of odorant adaptation in the olfactory receptor cell. Nature 385:677-679.

Kurahashi T, Shibuya T (1990) $\mathrm{Ca}^{2+}$-dependent adaptive properties in the solitary olfactory receptor cell of the newt. Brain Res 515:261-268.

Kurahashi T, Yau KW (1993) Co-existence of cationic and chloride compo- 
nents in odorant-induced current of vertebrate olfactory receptor cells. Nature 363:71-74.

Kurahashi T, Yau KW (1994) Olfactory transduction. Tale of an unusual chloride current. Curr Biol 4:118-120.

Larsson HP, Kleene SJ, Lecar H (1997) Noise analysis of ion channels in non-space-clamped cables: estimates of channel parameters in olfactory cilia. Biophys J 72:1193-1203.

Leinders-Zufall T, Rand MN, Shepherd GM, Greer CA, Zufall F (1997) Calcium entry through cyclic nucleotide-gated channels in individual cilia of olfactory receptor cells: spatiotemporal dynamics. J Neurosci 17:4136-4148.

Lindow MS, Menco BP (1984) Observation on axonemes and membranes of olfactory and respiratory cilia in frog and rats using tannic acidsupplemented fixation and photographic rotation. J Ultrastructure Res $86: 18-30$.

Lowe G, Gold GH (1991) The spatial distributions of odorant sensitivity and odorant-induced currents in salamander olfactory receptor cells. J Physiol (Lond) 442:147-168.

Lowe G, Gold GH (1993) Contribution of the ciliary cyclic nucleotide-gated conductance to olfactory transduction in the salamander. J Physiol (Lond) 462:175-196.

Lowe G, Gold GH (1995) Olfactory transduction is intrinsically noisy. Proc Natl Acad Sci USA 92:7864-7868.

Lowe G, Nakamura T, Gold GH (1989) Adenylate cyclase mediates olfactory transduction for a wide variety of odorants. Proc Natl Acad Sci USA 86:5641-5645.

Lynch JW, Barry PH (1989) Action potentials initiated by single channels opening in a small neuron (rat olfactory receptor). Biophys J 55:755-768.

Matsuzaki O, Bakin RE, Cai X, Menco BP, Ronnett GV (1999) Localization of the olfactory cyclic nucleotide-gated channel subunit 1 in normal, embryonic and regenerating olfactory epithelium. Neuroscience 94:131-140.
Menini A, Picco C, Firestein S (1995) Quantal-like current fluctuations induced by odorants in olfactory receptor cells. Nature 373:435-437.

Miwa N, Uebi T, Kawamura S (2000) Characterization of p26olf, a novel calcium-binding protein in the frog olfactory epithelium. J Biol Chem 275:27245-27249.

Pace U, Hanski E, Salomon Y, Lancet D (1985) Odorant-sensitive adenylate cyclase may mediate olfactory reception. Nature 316:225-258.

Reisert J, Bauer PJ, Yau KW, Frings S (2003) The Ca-activated Cl channel and its control in rat olfactory receptor neurons. J Gen Physiol 122:349-363.

Schild D, Restrepo D (1998) Transduction mechanisms in vertebrate olfactory receptor cells. Physiol Rev 78:429-466.

Schwarzenbacher K, Fleischer J, Breer H (2005) Formation and maturation of olfactory cilia monitored by odorant receptor-specific antibodies. Histochem Cell Biol 123:419-428.

Sklar PB, Anholt RR, Synder SH (1986) The odorant-sensitive adenylate cyclase of olfactory receptor cells. Differential stimulation by distinct classes of odorants. J Biol Chem 261:15538-15543.

Takeuchi H, Kurahashi T (2002) Photolysis of caged cyclic AMP in the ciliary cytoplasm of the newt olfactory receptor cell. J Physiol (Lond) 541:825-833.

Takeuchi H, Kurahashi T (2003) Identification of second messenger mediating signal transduction in the olfactory receptor cell. J Gen Physiol 122:557-567.

Takeuchi H, Kurahashi T (2005) Mechanism of signal amplification in the olfactory sensory cilia. J Neurosci 25:11084-11091.

Varnum MD, Zagotta WN (1996) Subunit interactions in the activation of cyclic nucleotide-gated ion channels. Biophys J 70:2667-2679.

Zheng J, Zagotta WN (2004) Stoichiometry and assembly of olfactory cyclic nucleotide-gated channels. Neuron 42:411-421.

Zufall F, Sheperd G, Firestein S (1991) Inhibition of the olfactory cyclic nucleotide gated ion channel by intracellular calcium. Proc Biol Sci 246: 225-230. 